\title{
A constraint-based programming approach to physical human-robot interaction
}

\author{
Gianni Borghesan, Bert Willaert and Joris De Schutter
}

\begin{abstract}
This work aims to extend the constraint-based formalism iTaSC for scenarios where physical human-robot interaction plays a central role, which is the case for e.g. surgical robotics, rehabilitation robotics and household robotics. To really exploit the potential of robots in these scenarios, it should be possible to enforce force and geometrical constraints in an easy and flexible way. iTaSC allows to express such constraints in different frames expressed in arbitrary spaces and to obtain control setpoints in a systematic way. In previous implementations of iTaSC, industrial velocity-controlled robots were considered. This work presents an extension of the iTaSCframework that allows to take advantage of the back-drivability of a robot thus avoiding the use of force sensors. Then, as a casestudy, the iTaSC-framework is used to formulate a (positionposition) teleoperation scheme. The theoretical findings are experimentally validated using a PR2 robot.
\end{abstract}

\section{INTRODUCTION AND MOTIVATIONS}

The last decade has seen a growing interest in robotic systems that can safely operate in poorly structured environments (where humans can be present) as well as robotic systems that directly interact with humans. Robots like the PR2, [1] and Justin [2], have been designed specifically to tackle these challenging scenarios. In the medical domain, examples are the use of telerobotic systems for surgery [3] and the use of robotic rehabilitation devices [4].

An important requirement for these systems is the ability to reliably control the physical interaction between the robot and the environment or the human. To really exploit the potential of such systems, however, it should also be possible to impose very different constraints, that the system complies with in an optimal way using the user's input as well as information about the environment obtained through different sensing modalities.

Some general constraints are given next together with examples from different application fields. The constraint can be i) to maintain a given pose w.r.t. to a moving object, in the surgical context this can be following a beating heart, [5], ii) to allow motion only along a specific trajectory, in the rehabilitation context this can be guiding a patient, [4], iii) to apply a specific force in a specific direction, in a household scenario, this can be to safely grasp a fragile object, [6], or perform a shared task with a person, [7], iv) to avoid collision with a specific (moving) object, in a industrial scenario this could be keeping the robot a minimum distance away from humans, [8], or v) to keep a force below a given threshold,

This work was partially supported by the PR2 beta program by Willow Garage.

G. Borghesan,B. Willaert and J. De Schutter are with the Department of Mechanical Engineering, K.U.Leuven, Heverlee, Belgium. name.surname@mech.kuleuven.be again in the surgical context this can be to avoid tissue damage, [9]. Next to these application constraints, there are also a number of system constraints that need to be taken into account, like the range of motion, force limits and velocity limits.

The description of these geometrical and force constraints in different frames and the derivation of the optimal control action, should be addressed by a constraint-based programming method of which iTaSC is a good example [8], [10]-[12]. iTaSC stands for instantaneous task specification using constraints, and could be seen as an extension of the Operational Space Formulation, [13], from which inherits some characteristics. The core of an iTaSC controller is the solver of a least-squares optimisation problem, that gives the desired joint velocities for the robot as output. The inputs for the iTaSC controller are (time-variable) setpoints for the constraints expressed in different frames. In case the problem is over-constrained (e.g. in case of conflicting constraints), the optimal solution of the solver depends on the implemented weighting factors.

Since the output of the iTaSC controller are robot joint velocities, its main application has been, till now, on velocitycontrolled robots, and interaction with the environment was mediated by force sensing (e.g. the human robot comanipulation task described in [11]). In many scenarios described above, however, low-impedance type robots are preferred in order to have a safe physical interaction between the robot and the human/environment. Since such robots also allow the estimation of contact forces, the use of a force sensor is not mandatory, which limits the cost of the device, increases the robustness, and avoids possible issues with the force sensor design (e.g. miniaturization and sterilization issues with surgical robotics).

The first contribution of this work is to extend the iTaSCframework to allow controlled physical interaction of a robot with an environment or a human operator without using a force sensor. To realize this successfully, the robot has to be position-controlled and sufficiently backdrivable, i.e. forces applied by the environment or by a human operator should result in a pose of the robot that is noticeably different from the desired pose that is the input for the robot's position controller.

The second contribution of this work is the formulation of a classical (position-position) teleoperation scheme as a casestudy for the iTaSC-framework and the above mentioned extension. Furthermore, it will be shown how easily a virtual fixture can be embedded in the control of the slave robot, revealing the potential of the iTaSC-framework. 
Experimental validation is carried out on the PR2 robot with one of its arms as the master device and the other arm as the slave device. This work has to be situated in the framework of the PR2 beta program, which aims a.o. at exploring the full potential of iTaSC for robotic systems operating in a human-shared environment.

\section{General Approach Description}

This section first describes in general terms how a problem is formulated in the iTaSC-framework. Next, it describes how the framework is extended to allow backdrivability of a position-controlled robot (without using a force sensor) and at last it describes how the iTaSC-framework can be used to create a teleoperation system.

\section{A. iTaSC basics}

The input for the iTaSC controller is a set of constraints on controllable variables. Referring to the examples in the introduction, these controllable variables can be a distance or a force between the robot and an object or the position of an object in a camera image. The output of the iTaSC controller is the desired joint velocities for the robotic system to be controlled. These joint velocities result in an optimal behaviour w.r.t. all the constraints. To resolve either redundancy or conflicting constraints, an objective function has to be set that defines this optimal behaviour.

To use the iTaSC-framework a constraint has to be defined via a kinematic loop relating two object frames (denoted with $o 1$ and $o 2$ ) with one of these object frames being attached to the robotic system to be controlled and one being attached to an object of interest. All kinematic loops, i.e. one for each constraint, are closed via the world frame.

To facilitate the description of the degrees of freedom between each two objects two intermediate feature frames (denoted by $f 1$ and $f 2$ ) can be used. These feature frames are not necessarily rigidly attached to their respective object. By defining these different object and feature frames, the iTaSC-framework allows to define constraints directly in one of these object or feature frames which is typically more intuitive/straightforward than having to define all constraints in the world frame.

\section{B. Backdrivability}

In previous iTaSC implementations, it is assumed that the robot behaves as an ideal velocity-controlled robot. Such kind of robot, however, cannot provide any information about the interaction forces with the environment and/or human operator if no force sensor is used. Here, the robot is assumed to be of the low-impedance type, gravity compensated and position-controlled. To make such a position-controlled robot backdrivable, ideally, the position set-point for the position controller should be continuously the same as the actual position of the robot.

To facilitate this, the use of a simulated robot is proposed here. The simulated robot, governed by the iTaSC controller, replaces the ideal velocity-controlled robot of the previous iTaSC implementations and thus acts as a pure integrator w.r.t



Fig. 1. Top: schematic representation of one of the two teleoperation stations, which exchange, through the communication channels, the simulated robot e.e. poses $o 2$ and $o 1 a$. Bottom: details of the joint controller, the real robot, the simulated robot (an integrator of desired joint velocities), and forward kinematics.

the commanded joint velocities. Furthermore, it has the same kinematic structure as the real robot. Now, being positioncontrolled, this real robot has its motor torques $\tau$ defined by the following proportional law:

$$
\boldsymbol{\tau}=\boldsymbol{K}_{\boldsymbol{q}}\left(\boldsymbol{q}-\boldsymbol{q}_{r}\right)
$$

with $\boldsymbol{q}$ and $\boldsymbol{q}_{r}$ being the joint angle vectors of respectively the simulated and the real robot. The matrix $\boldsymbol{K}_{q}$ is typically a diagonal gain matrix. The vector $\boldsymbol{q}$ is obtained by applying the desired joint velocities $\dot{\boldsymbol{q}}$, which is the output of the iTaSC controller, to the simulated robot. Thus, $\boldsymbol{q}$ is obtained by integrating $\dot{\boldsymbol{q}}$. At last, applying the forward kinematics to $\boldsymbol{q}$ and $\boldsymbol{q}_{r}$, gives the poses of the end-effector of respectively the simulated $(o 2)$ and the real robot $(o 1 b)$, which serve as inputs for the iTaSC controller.

It is important to see that the iTaSC controller does not directly control the real robot, but uses the real robot end effector pose to obtain an estimate for the interaction forces which, in turn, are used by the iTaSC controller to regulate the simulated robot. Stated differently, the back-driveability of the real robot is obtained by constraining the simulated robot to track the real robot's end-effector pose. The above described flow of events is illustrated in Fig. 1. Note that $\boldsymbol{y}_{d}$ stands for the desired outcome for the iTaSC controller which will become clear in the next section.

\section{Teleoperation}

In order to create a teleoperation system, two independent iTaSC controllers are used. Both the simulated robot at the master side and at the simulated robot at the slave side have their own iTaSC controller and they are constrained to track both the real robot at their respective side (see the previous subsection) and the simulated robot at the other side. Thus each of the two iTaSC controller needs as inputs: 


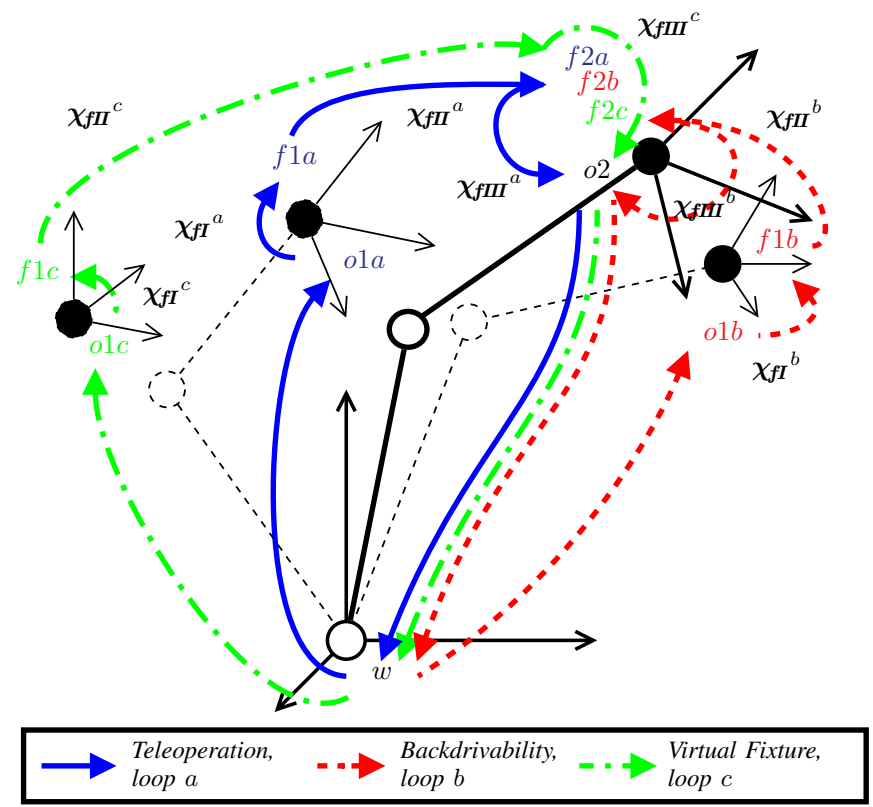

Fig. 2. The three kinematic loops with object and feature frames. The solid line represents the simulated robot, with the object frame $o 2$ attached to its end effector. The dashed line on the right represents the real robot and the one on the left represents the other simulated robot, with respectively the object frames $o 1 b$ and $o 1 a$ attached to their end effectors. The object frame $o 1 c$ is attached to the object that defines the virtual fixture.

i) the real robot end-effector pose at its respective side, $(o 1 b)$, ii) the simulated robot end-effector pose $(o 2)$, iii) the endeffector pose of the other simulated robot $(o 1 a)$, and iv) the desired outcome $\boldsymbol{y}_{d}$. This teleoperation scheme behaves like a classical position-position scheme since the two simulated robots are constrained to track each other. Finally, both the simulated robots could be further constrained to track other objects $(o c 1, o d 1, \ldots)$, which is how virtual fixtures can be defined.

\section{SYNTHESIS OF THE ITASC CONTROLLER}

This section addresses the synthesis of the iTaSC controller for both the simulated master and the slave robot, following the methodology described in [11] and adding details only where needed.

The control is achieved through four steps: i) first of all, the geometric relations (Sec. III-A), ii) and their relative motions (Sec. III-B) must be described. iii) Then, the constraints are defined (Sec. III-C), and lastly, iv) the way to impose such constraints is addressed (Sec. III-D).

\section{A. Choice of objects and features}

The first step is to define the three kinematics loops, by choosing the frames in which the constraints will be expressed, i.e. the features frames $f 1 i$ and $f 2 i$, and relate these frames to the object frames $o 1 i$ and $o 2$. As defined in the previous section, the backdrivability loop (loop $b$ ) relates the object frame of the simulated robot $o 2$ to the object frame of the real robot $o 1 b$. The teleoperation loop (loop $a$ ) relates the object frame of the simulated robot $o 2$ to the object frame of the other simulated robot $o 1 a$ and the virtual fixture loop (loop $c$ ) relates the object frame of the simulated robot $o 2$ to the object frame of a arbitrary object. For all loops the world reference frame $w$ coincides with the base of the robot. A schematic representation of how frames are located is reported in Fig. 2.

For each loop, degrees of freedom between frames must be assigned, and feature coordinates must be chosen. Since here each constraint can be most easily expressed in one reference frame, the feature frames have been defined to have the same pose as their respective object frames $(f 1 i=o 1 i$, $f 2 i=o 2$ for loops $i=a, b, c)$. Stated differently, all six degrees of freedom occur between $f 1 a$ and $f 2 a, f 1 b$ and $f 2 b$ and between $f 1 c$ and $f 2 c$. Next, these degrees of freedom are represented by the vectors of feature coordinates $\chi_{f I I}{ }^{a}$, $\chi_{\boldsymbol{f I I}}{ }^{b}$ and $\chi_{\boldsymbol{f I I}}{ }^{c}$ :

$$
\begin{aligned}
\chi_{\boldsymbol{f I I}}{ }^{a} & =\left(x^{a}, y^{a}, z^{a}, \varphi^{a}, \vartheta^{a}, \psi^{a}\right)^{T}, \\
\chi_{\boldsymbol{f I I}}{ }^{b} & =\left(x^{b}, y^{b}, z^{b}, \varphi^{b}, \vartheta^{b}, \psi^{b}\right)^{T}, \\
\chi_{\boldsymbol{f I I}}{ }^{c} & =\left(x^{c}, y^{c}, z^{c}, \varphi^{c}, \vartheta^{c}, \psi^{c}\right)^{T},
\end{aligned}
$$

where $x^{i}, y^{i}, z^{i}$ are the position and $\varphi^{i}, \vartheta^{i}, \psi^{i}$ the roll pitch yaw angles of the object frame $o 2$ expressed w.r.t. the object frame $o 1 i$.

\section{B. Modeling of the relative motion}

The relative motions of the different frames are modeled by means of twists $\mathbf{t}$ :

$$
\begin{aligned}
\mathbf{t}_{o 1 i}^{f 1 i} & =\boldsymbol{J}_{\boldsymbol{f I}}^{i} \dot{\chi}_{\boldsymbol{f I}}{ }^{i}=\boldsymbol{O}, \\
\mathbf{t}_{f 1 i}^{f 2 i} & =\boldsymbol{J}_{f I I}^{i} \dot{\chi}_{f I I}^{i}, \\
\mathbf{t}_{f 2 i}^{o 2} & =\boldsymbol{J}_{f I I}^{i} \dot{\chi}_{f I I I}{ }^{i}=\boldsymbol{O} .
\end{aligned} \quad i=a, b, c
$$

Since the feature coordinates $\chi_{f I I}{ }^{i}$ are expressed in minimal coordinates, the feature Jacobians $J_{f I I}^{i}$ that map the time derivative of $\chi_{f I I}{ }^{i}$ to the twist $\mathbf{t}_{f 1 i}^{f 2 i}$ must include an integrating factor:

$$
\boldsymbol{J}_{f I I}^{i}=\left[\begin{array}{ccc}
\boldsymbol{1}_{3 \times 3} & \multicolumn{3}{c}{\boldsymbol{O}_{3 \times 3}} \\
\boldsymbol{O}_{3 \times 3} & {\left[\begin{array}{ccc}
0 & -\sin \left(\varphi^{i}\right) & \cos \left(\vartheta^{i}\right) \cos \left(\varphi^{i}\right) \\
0 & \cos \left(\varphi^{i}\right) & \left.\cos \left(\vartheta^{i}\right) \sin \varphi^{i}\right) \\
1 & 0 & -\sin \left(\varphi^{i}\right)
\end{array}\right]}
\end{array}\right]
$$

Furthermore, the feature Jacobians $J_{f I}^{i}$ and $J_{f I I I}^{i}$ are not defined, since $\dot{\chi}_{f I}{ }^{i}$ and $\dot{\chi}_{f I I I}{ }^{i}$ are zero-dimension vectors.

The feature Jacobian $\boldsymbol{J}_{f}^{i}$ is the union of the single feature Jacobians, expressed in the world frame:

$$
\boldsymbol{J}_{\boldsymbol{f}}^{i}=\left[{ }_{w} \boldsymbol{J}_{\boldsymbol{f I}}^{i}\left|{ }_{w} \boldsymbol{J}_{\boldsymbol{f I I}}^{i}\right|{ }_{w} \boldsymbol{J}_{\boldsymbol{f I I I}}^{i}\right]={ }_{w} \boldsymbol{J}_{\boldsymbol{f I I}}^{i}
$$

The single feature Jacobians can be expressed in the world frame by making use of the adjoint matrices:

$$
{ }_{w} \boldsymbol{J}_{\boldsymbol{f I}}^{i}=\left[\begin{array}{cc}
\boldsymbol{R}_{w}^{f 1 i} & \boldsymbol{p}_{w}^{f 2 i} \\
\boldsymbol{O}_{3 \times 3} & \boldsymbol{R}_{w}^{f 1 i}
\end{array}\right] \boldsymbol{J}_{\boldsymbol{f I I}}^{i},
$$

where $\boldsymbol{R}_{w}^{j}$ is the rotation matrix from the world frame $w$ to frame $j$, and $\boldsymbol{p}_{w}^{j}$ is the skew symmetric matrix built with the position vector of frame $j$ w.r.t the world frame $w$. By 
considering the closure of the kinematic loop at a velocity level, the following holds:

$$
\mathbf{t}_{o 1 i}^{o 2}=\mathbf{t}_{o 1 i}^{f 1 i}+\mathbf{t}_{f 1 i}^{f 2 i}+\mathbf{t}_{f 2 i}^{o 2} \quad \Rightarrow \quad{ }_{w} \mathbf{t}_{o 1 i}^{o 2}=J_{f}^{i} \dot{\chi}_{\boldsymbol{f}}^{i}
$$

where ${ }_{w} \mathbf{t}_{o 1 i}^{o 2}$ is the twist of $o 2$ w.r.t. $o 1 i$, expressed in the world frame, and $\dot{\chi}_{f}{ }^{i}$ is defined as follows:

$$
\dot{\chi}_{\boldsymbol{f}}{ }^{i}=\left[\left(\dot{\chi}_{f I}{ }^{i}\right)^{T}\left|\left(\dot{\chi}_{f I I}{ }^{i}\right)^{T}\right|\left(\dot{\chi}_{f I I I}\right)^{T}\right]^{T}=\dot{\chi}_{f I I}{ }^{i}
$$

On the other hand, the twist ${ }_{w} \mathbf{t}_{o 2}^{o 1 i}$ can also be written as:

$$
\begin{aligned}
{ }_{w} \mathbf{t}_{o 2}^{o 1 i} & ={ }_{w} \mathbf{t}_{w}^{o 1 i}-{ }_{w} \mathbf{t}_{w}{ }^{22}, \\
{ }_{w} \mathbf{t}_{w}^{o 1 i} & =\boldsymbol{J}_{r 1}^{i} \dot{\boldsymbol{q}}+\mathbf{t}_{u 1 i}, \\
{ }_{w} \mathbf{t}_{w}^{o 2} & =\boldsymbol{J}_{\boldsymbol{r} 2} \dot{\boldsymbol{q}}+\mathbf{t}_{u 2} .
\end{aligned}
$$

Here, ${ }_{w} \mathbf{t}_{w}^{\mathbf{t} j(i)}$ is the twist of the object frame oj(i) expressed in the world frame. Each of these object twists can be divided in a controlled part, $\boldsymbol{J}_{\boldsymbol{r} j}^{(i)} \dot{\boldsymbol{q}}$, and an uncontrolled part, $\mathbf{t}_{u j(i)}$, where $J_{\boldsymbol{r} j}^{(i)}$ is the Jacobian that maps the joint velocities of the simulated robot $\dot{\boldsymbol{q}}$ to ${ }_{w} \mathbf{t}_{w}^{\mathrm{oj}(i)}$, while $\mathbf{t}_{u j(i)}$ is the uncontrolled twist of each object. It is important to understand that both at the master and at the slave side there is an independent iTaSC controller that only controls the corresponding simulated robot. As shown also in Fig. 1, the only output of the iTaSC controller is $\dot{\boldsymbol{q}}$, which directly controls all the degrees of freedom of the simulated robot at its respective side $\left(\mathbf{t}_{u 2}=0\right)$, but controls neither the twist of the end-effector of the real robot at this side $\left(\boldsymbol{J}_{\boldsymbol{r} 1}^{b}\right.$ cannot be defined), nor the twist of the simulated robot at the other side ( $\boldsymbol{J}_{\boldsymbol{r} 1}^{a}$ cannot be defined):

$$
\begin{gathered}
{ }_{w} \mathbf{t}_{o 2}^{o 1 i}={ }_{w} \mathbf{t}_{w}^{o 1 i}-{ }_{w} \mathbf{t}_{w}^{o 2}, \\
{ }_{w} \mathbf{t}_{w}^{o 1 i}=\mathbf{t}_{u 1 i}, \\
{ }_{w} \mathbf{t}_{w}^{22}=\boldsymbol{J}_{\boldsymbol{r} 2} \dot{\boldsymbol{q}} .
\end{gathered}
$$

Now combining the two velocity closure loop equations results in:

$$
\boldsymbol{J}_{\boldsymbol{r}}^{i} \dot{\boldsymbol{q}}+\boldsymbol{J}_{\boldsymbol{f}}^{i} \dot{\chi}_{\boldsymbol{f}}{ }^{i}+\mathbf{t}_{u}^{i}=0, \quad \boldsymbol{J}_{\boldsymbol{r}}^{i}=-\boldsymbol{J}_{\boldsymbol{r} 2}^{i}, \quad \mathbf{t}_{u}^{i}=\mathbf{t}_{u 1 i}
$$

To insert the loop $c$, the same derivations for loop $a$ applies, optionally considering $o 1 c$ as fixed $\left(\mathbf{t}_{\text {uic }}=\boldsymbol{0}\right)$.

\section{Definition of output and constraints}

In iTaSC, constraints are defined on output $\boldsymbol{y}$, by making them follow a desired value or trajectory $\boldsymbol{y}_{d}$. Output can be any (linear) combination of feature coordinates $\chi_{f}$ and joint angles. Normally only a selection is made, by shaping the selection matrices $C_{f}^{i}$ and $C_{q}^{i}$.

$$
\left[\begin{array}{ll}
C_{q}^{i} & C_{f}^{i}
\end{array}\right]\left[\begin{array}{c}
\dot{q}^{i} \\
\dot{\chi}_{f}^{i}
\end{array}\right]=\dot{y}^{i}
$$

In this case, not considering the virtual fixture:

$$
\boldsymbol{y}^{i}=\chi_{f I I}{ }^{i}, \quad C_{f}^{i}=1_{6 \times 6}, \quad i=a, b
$$

and $C_{q}^{i}$ is not defined, since no constraint is expressed in the joint space.
The constraints are enforced by computing a desired velocity $\dot{\boldsymbol{y}}^{i}$; the simplest way to compute desired velocities is a proportional law:

$$
\dot{\boldsymbol{y}}^{i}=\boldsymbol{K}_{p}^{i}\left(\boldsymbol{y}_{d}^{i}-\boldsymbol{y}^{i}\right),
$$

where $\boldsymbol{K}_{p}^{i}$ is a gain matrix.

In the case of the slave system, other outputs for other constraints (i.e. the virtual fixture) must be added, in a number equal to the constrained directions (here five other directions will be constrained).

Once outputs and constraints are defined, the desired outputs $\boldsymbol{y}_{d}$ must be given. In this case, the desired outputs values are all zero, representing the goal to force $f 2 i$ to coincide to $f 1 i$, and consequently $o 2$ with $o 1 i$, in all loops.

\section{The instantaneous motion problem}

Once the desired velocities $\dot{\boldsymbol{y}}^{i}$ are computed, then the method to derive the desired joint velocities must be addressed. In iTaSC, the joint velocities computation is formulated as an optimization problem.

In order to correctly express the instantaneous motion problem, the following quantities must be defined:

$$
\begin{gathered}
\boldsymbol{A}=\boldsymbol{C}_{\boldsymbol{q}}-\boldsymbol{C}_{\boldsymbol{f}} \boldsymbol{J}_{\boldsymbol{f}}^{-1} \boldsymbol{J}_{\boldsymbol{r}}, \quad \boldsymbol{B}=\boldsymbol{C}_{\boldsymbol{f}} \boldsymbol{J}_{\boldsymbol{f}}^{-1}, \\
\boldsymbol{C}_{\boldsymbol{f}}=\operatorname{diag}\left(\boldsymbol{C}_{\boldsymbol{f}}^{a}, \boldsymbol{C}_{\boldsymbol{f}}^{b}\right), \quad \boldsymbol{J}_{\boldsymbol{f}}=\operatorname{diag}\left(\boldsymbol{J}_{\boldsymbol{f}}^{a}, \boldsymbol{J}_{\boldsymbol{f}}^{b}\right), \\
\boldsymbol{C}_{\boldsymbol{q}}=\left[\begin{array}{l}
\boldsymbol{C}_{\boldsymbol{q}}^{a} \\
\boldsymbol{C}_{\boldsymbol{q}}^{b}
\end{array}\right], \boldsymbol{J}_{\boldsymbol{r}}=\left[\begin{array}{l}
\boldsymbol{J}_{\boldsymbol{r}}^{a} \\
\boldsymbol{J}_{\boldsymbol{r}}^{b}
\end{array}\right], \boldsymbol{\boldsymbol { y }}=\left[\begin{array}{c}
\dot{\boldsymbol{y}}^{a} \\
\dot{\boldsymbol{y}}^{b}
\end{array}\right], \mathbf{t}_{u}=\left[\begin{array}{l}
\mathbf{t}_{u}^{a} \\
\mathbf{t}_{u}^{b}
\end{array}\right]
\end{gathered}
$$

Definitions expressed in (11) hold for the master robot, while, for the slave robot, also the matrices corresponding loop $c$ must be taken into account. The problem can now be expressed as an optimization problem:

$$
\min _{\dot{\boldsymbol{q}}}\left\|\boldsymbol{A} \dot{\boldsymbol{q}}-\dot{\boldsymbol{y}}-\boldsymbol{B} \mathbf{t}_{u}\right\|_{W}
$$

In the case at hand, the system is overconstrained, and a weighting matrix $\boldsymbol{W}$ has been introduced to describe the prominence of a constraint over others. Here, the solution of this problem is achieved by means of the Moore-Penrose pseudoinverse, but other alternatives are available (as reported in [10]).

\section{System BehaVIOUR AND TUNING}

This section briefly illustrates how the system behaves and how gains and weights of the iTaSC controller influence such behaviour.

\section{A. Behaviour imposed by the backdrivability loop}

First of all, we investigate how the simulated robot behaves w.r.t. external forces. To do so, only the backdrivability constraint is considered (i.e. quantities referring to loop $a$ are not defined); in this case, the optimization problem is exactly constrained and (12) has a unique solution. By substitution of $\boldsymbol{J}_{\boldsymbol{r} 2} \dot{\boldsymbol{q}}={ }_{w} \mathbf{t}_{w} \mathrm{t}_{w}$ (derived by (7)), and imposing a finite displacement to the end effector $\delta \boldsymbol{y}(o 2-o 1 b$ expressed in $o 1 b$ ), by means of the output equation (10), the following holds: 


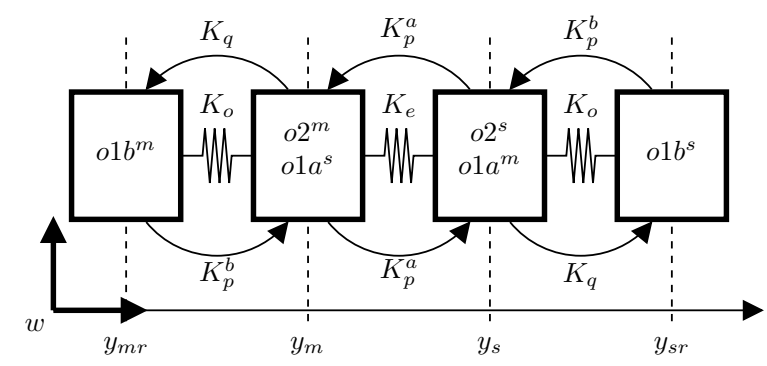

Fig. 3. Equivalent representation of a 1 dof teleoperation system, at the equilibrium, the system behaves as a series of springs, which stiffness depend by the controller parameters.

$$
\begin{aligned}
\boldsymbol{J}_{\boldsymbol{f}}{ }^{-1}{ }_{w} \mathbf{t}_{w}{ }^{2} & =\boldsymbol{K}_{p} \delta \boldsymbol{y}+\boldsymbol{J}_{\boldsymbol{f}}{ }^{-1} \mathbf{t}_{u} \\
\boldsymbol{J}_{\boldsymbol{f}}{ }^{-1}{ }_{w} \mathbf{t}_{w} 2 & =\boldsymbol{K}_{p} \boldsymbol{J}_{\boldsymbol{f}}{ }^{-1} \delta \boldsymbol{y}_{w}+\boldsymbol{J}_{\boldsymbol{f}}{ }^{-1} \mathbf{t}_{u} \\
\Rightarrow_{w} \mathbf{t}_{w}{ }^{2} & =\boldsymbol{J}_{\boldsymbol{f}} \boldsymbol{K}_{p} \boldsymbol{J}_{\boldsymbol{f}}{ }^{-1} \delta \boldsymbol{y}_{w}+\mathbf{t}_{u}
\end{aligned}
$$

where $\delta \boldsymbol{y}_{w}$ denotes the world-expressed finite displacement.

Equation (13) highlights that in the ideal case, the simulated robot follows the real one exactly because of the feedforward term (since $\mathbf{t}_{u}$ is the twist of the real robot end effector), since $\delta \boldsymbol{y}$ is negligible.

In practice, the simulated robot lags behind the real one, due to delays introduced by discrete-time control and inaccurate robot velocity estimation, and some error accumulates, resulting in the low-level control exerting a torque $\tau$. In quasi-static hypothesis, the external wrench $\boldsymbol{w}_{e}$ is derived by $\tau$ (assuming quasi-static conditions), and the finite displacement is related to the wrench, by:

$$
\delta \boldsymbol{y}_{w}=\boldsymbol{J}_{\boldsymbol{q}} \delta \boldsymbol{q}=\boldsymbol{J}_{\boldsymbol{q} r} \boldsymbol{K}_{\boldsymbol{q}}{ }^{-1} \boldsymbol{\tau} \approx \boldsymbol{J}_{\boldsymbol{q} r} \boldsymbol{K}_{\boldsymbol{q}}{ }^{-1} \boldsymbol{J}_{\boldsymbol{q} r}^{T} \boldsymbol{w}_{e}
$$

By combining (13), and (14), and supposing that $\delta \boldsymbol{q}$ is sufficiently small $\left(J_{\boldsymbol{q}} \approx J_{\boldsymbol{q}}\right)$, it is possible to compute the relation between external wrenches and simulated robot velocity:

$$
{ }_{w} \mathbf{t}_{w}^{o 2} \approx \boldsymbol{J}_{\boldsymbol{f}} \boldsymbol{K}_{p} \boldsymbol{J}_{\boldsymbol{f}}^{-1} \boldsymbol{J}_{\boldsymbol{q} r} \boldsymbol{K}_{\boldsymbol{q}}{ }^{-1} \boldsymbol{J}_{\boldsymbol{q} r}^{T} \boldsymbol{w}_{e}+\mathbf{t}_{u}
$$

Since $\boldsymbol{K}_{p}$ can be chosen freely, it is possible to assign desired dynamics to the simulated robot in the operational space, as expressed by:

$$
{ }_{w} \mathbf{t}_{w}^{o 2}=\overline{\boldsymbol{K}_{p}} \boldsymbol{w}_{e}+\mathbf{t}_{u},
$$

where $\overline{\boldsymbol{K}_{p}}$ is an arbitrary matrix, a good choice for $\overline{\boldsymbol{K}_{p}}$ is a diagonal matrix, so that the various directions of motion become decoupled. To achieve such dynamics, $\boldsymbol{K}_{p}$ must be computed on line at each iteration step:

$$
\boldsymbol{K}_{p}=\boldsymbol{J}_{\boldsymbol{f}}^{-1} \overline{\boldsymbol{K}_{p}}\left(\boldsymbol{J}_{\boldsymbol{q} r} \boldsymbol{K}_{\boldsymbol{q}}{ }^{-1} \boldsymbol{J}_{\boldsymbol{q} r}^{T}\right)^{-1} \boldsymbol{J}_{\boldsymbol{f}}=\boldsymbol{J}_{\boldsymbol{f}}^{-1} \overline{\boldsymbol{K}_{p}} \boldsymbol{K}_{\boldsymbol{o}} \boldsymbol{J}_{\boldsymbol{f}}
$$

where $\boldsymbol{K}_{\boldsymbol{O}}$ is operational stiffness defined as:

$$
\boldsymbol{K}_{\boldsymbol{o}}=\boldsymbol{J}_{\boldsymbol{q} r}^{-T} \boldsymbol{K}_{\boldsymbol{q}} \boldsymbol{J}_{\boldsymbol{q} r}^{-1}
$$

Since $\overline{\boldsymbol{K}_{p}}$ relates forces to velocities, it has the dimensions of an inverse damping.

\section{B. Static behaviour of the teleoperation system}

In order to illustrate also the behaviour of the teleoperation system subject to both the loop $a$ and $b$, let us consider only a one degree-of-freedom system (e.g. the $y$-axis), subject to backdrivability and teleoperation constraints, as depicted in Fig. 3. Let $y_{m}, y_{s}$ and $y_{m r}, y_{s r}$ be the displacements of the simulated and real robots w.r.t the world reference for master $(m)$ and slave $(s)$. If the real robots are kept motionless (and subject to external forces $f_{m}, f_{s}$ ), and the simulated robots are governed by iTaSC controllers imposing teleoperation and backdrivability constraints, weighted with the matrix $\boldsymbol{W}=\operatorname{diag}\left[w_{a}, w_{b}\right]$, then, the equations governing the master and slave simulated robots are:

$$
\begin{aligned}
\dot{y}_{m} & =\frac{\left(K_{p}^{a}\left(y_{s}-y_{m}\right)+\dot{y}_{s}\right) w_{a}^{2}+\left(K_{p}^{b}\left(y_{m r}-y_{m}\right)+\dot{y}_{m r}\right) w_{b}^{2}}{w_{a}^{2}+w_{b}^{2}} \\
& =\frac{\left(K_{p}^{a}\left(y_{s}-y_{m}\right)+\dot{y}_{s}\right) w_{a}^{2}+\left(-\overline{K_{p}^{b}} f_{m}+\dot{y}_{m r}\right) w_{b}^{2}}{w_{a}^{2}+w_{b}^{2}}, \\
\dot{y}_{s} & =\frac{\left(K_{p}^{a}\left(y_{m}-y_{s}\right)+\dot{y}_{m}\right) w_{a}^{2}+\left(-\overline{K_{p}^{b}} f_{s}+\dot{y}_{s r}\right) w_{b}^{2}}{w_{a}^{2}+w_{b}^{2}}
\end{aligned}
$$

In static conditions $\left(\dot{y}_{m}=0, \dot{y}_{s}=0, \dot{y}_{m r}=0\right.$, and $\dot{y}_{s r}=0$ ), it is possible to determine the stiffnesses that govern the settling positions of the real and simulated robot. The stiffness between the simulated robots is:

$$
K_{e}=\frac{f_{m}}{y_{s}-y_{m}}=\frac{f_{s}}{y_{m}-y_{s}}=\frac{K_{p}^{a} w_{a}^{2}}{\overline{K_{p}^{b}} w_{b}^{2}}
$$

The real robots are commanded by the joint controllers, so the stiffness between each of them and the corresponding simulated robot is governed by (18).

Fig. 3 shows how each part of the system is influenced by the other, with curved arrows; for example, the behaviour of the master simulated robot $\left(o 2^{m}\right)$ is influenced by both the master real and the slave simulated robots $\left(o 1 b^{m}\right.$ and $\left.o 1^{a}\right)$, while the master real robot $\left(o 1 b^{m}\right)$ is influenced only by the simulated robot $\left(o 2^{m}\right)$ by means of the low level controller ( $\boldsymbol{K}_{\boldsymbol{o}}$ in operational space). Spring symbols represents the behaviour in static conditions. From (20) and (18), it is possible to tune the system w.r.t. the static behaviour. Other aspects, omitted for sake of brevity, can be characterized, e.g. the settling time toward one or other constraints.

\section{EXPERIMENTAL RESULTS}

Experiments have been carried out on the PR2, whose arms have been used as a master-slave teleoperation platform. The control system has been realized employing ROS, [14], (to access the PR2 controllers) and OROCOS, [15], where the iTaSC controllers are implemented. The two PR2 arms are controlled by a joint controller that receives the joint angle set points and publishes the joint measurements at $100 \mathrm{~Hz}$. A set of three experiments is reported, highlighting the positions of master and slave end-effectors computed with the real angles, (defined as "m.r." and "s.r." respectively), and the positions of master and slave obtained from the joint angles of the simulated robot ("m.s." and "s.s."). 


\begin{tabular}{|c|c|}
\hline Parameter & diagonal elements \\
\hline$K_{q}$ & $\left(\begin{array}{lllllll}240 & 120 & 100 & 70 & 30 & 30 & 30\end{array}\right)[\mathrm{Nm} / \mathrm{rad}]$ \\
\hline $\boldsymbol{K}_{p}{ }^{a}$ & $\left(\begin{array}{llllll}20 & 20 & 20 & 20 & 20 & 20\end{array}\right)[1 / \mathrm{s}]$ \\
\hline$\overline{\boldsymbol{K}_{p}{ }^{b}}$ & $\left(1_{3 \times 3} \cdot 0.05[\mathrm{~m} / \mathrm{sN}] \quad 1_{3 \times 3} \cdot 0.1[\mathrm{rad} / \mathrm{sN}]\right)$ \\
\hline $\boldsymbol{K}_{p}^{c}$ & $\left(\begin{array}{llllll}1 & 1 & 1 & 1 & 1 & 1\end{array}\right)[1 / \mathrm{s}]$ \\
\hline$W$ & $\left(\begin{array}{lll}\mathbf{1}_{6 \times 6} & \mathbf{1}_{6 \times 6} & 35 \cdot 1_{6 \times 6}\end{array}\right)[\cdot]$ \\
\hline
\end{tabular}

TABLE I

NUMERICAL VALUES OF MOST RELEVANT PARAMETERS.

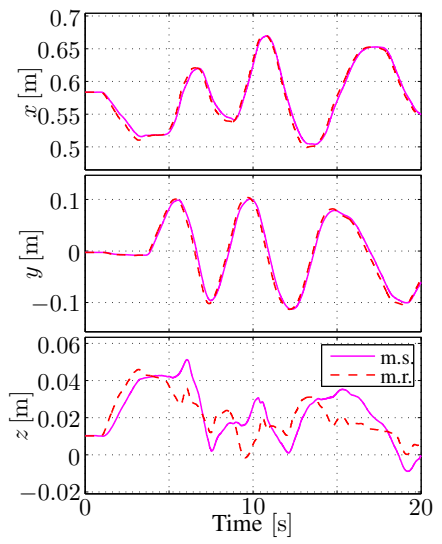

(a) Position

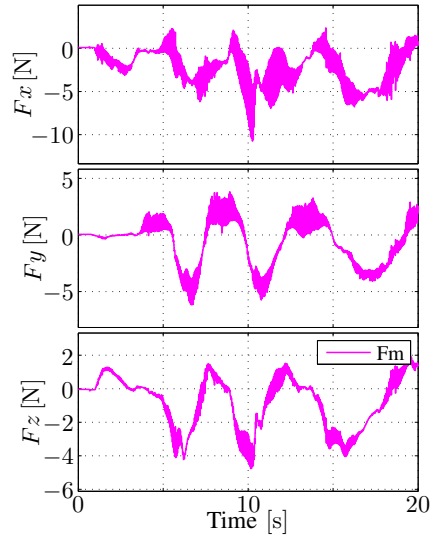

(b) Force
Fig. 4. Master robot driven with backdrivability loop only (no teleoperation).

The orientations have been omitted for sake of brevity. Along with the positions, the forces commanded to the master robot and slave robot (with inverted sign for comparative purpose), derived from actuation joint torques, are reported. Numerical values of gains and weight are reported in Table I.

Fig. 4 represents the behaviour of the master robot subject only to the backdrivability loop, (no teleoperation occurs). It can be seen how the simulated robot follows the real one when the user moves the robot, enforcing (16); in particular it is seen how the joint controller generates a force (opposing the user's motion) when the simulated robot position is not updated promptly enough. This limited tracking behaviour can be explained as a direct and indirect effect of the low sampling frequency of the PR2 control interface. The indirect effects refers to the fact that it restricts the gain $\boldsymbol{K}_{p}$.

Fig. 5 reports an experiment of pure teleoperation. In the first $30 \mathrm{~s}$, the slave robot moves in free space. As the user moves the master robot (approximately making circles in the $x-y$ plane), the movement is transmitted to the master simulated robot, and then to the simulated and real slave robots. This behaviour is evident when looking at the trend of the four tracks in the $x$ direction, at ten seconds, as they follow each other in the described order. After $30 \mathrm{~s}$, contact takes place with an object that is located at approximately $x=0.58 \mathrm{~m}$. The position now reached by each robot depends on the different gains of the iTaSC controllers and low level controllers, as depicted by Fig. 3 .

No big difference in the force following aspects has been noticed between contact and free-space, apart that force reflection is more faithful when the robots are motionless or slowly moving. Note that the fairly large forces in free space can be explained by the considerable dynamics of the PR2-arm. Due to these dynamics and with a position-position like control scheme, the contrast between the feeling in free space and the feeling when contacting an object is limited for this platform.

During the experiment reported in Fig. 6, a virtual fixture is employed; which is shown as the thick grey line in the position graphs and labelled as "v.f.". The virtual fixture constrains all but the $x$ direction, and for such reason its $x$ component is not shown. As before, this experiment is divided in two parts: in the first 50 seconds, the user moves the master robot so that the slave does not touch the obstacle already present in the previous experiment, trying to execute circular movements, then contact happens. In the first part, the slave robot is free to move along the $x$ direction, while movements in the other directions are restricted by the virtual fixture, and the slave simulated robot practically does not move. Since no external force is applied to the slave robot, very small deviations of the slave robot w.r.t. the virtual fixture are registered. At the master side, the $y$ movements of the user are opposed by the robot, as the master simulated robot position is defined by a weighted combination of the slave simulated robot position (practically fixed on the v.f.), and the real robot position.

After $50 \mathrm{~s}$ contact occurs. As the virtual fixture does not hinder the movements along the $x$ direction, the same discussion of the previous case applies. In this case, the master and slave force in the $y$-direction are very different. From the point of view of the user, the nature of the constraint at the slave side (real or virtual) is not discernible by means of the force only.

\section{CONCLUSION}

This work describes how the iTaSC approach can be expanded to control backdrivable devices, without the employment of force sensing device. This extension allows to broaden the field of employment of iTaSC toward physical human-robot interaction applications. As example of such applications, a traditional teleoperation scheme has been implemented and experimentally tested on the PR2 robot. Moreover, a proof-of-concept shows how a virtual fixture feature can easily be integrated into the controller, showing the potential of iTaSC for such applications. It is important to understand that, although a simple virtual fixture has been implemented, the same methodology applies to any kind of geometrical or force constraints. The physical behaviour of the system is briefly discussed, along with some guidelines on the choice of gains and weights of the controller, and how they influence the behaviour of the system. 


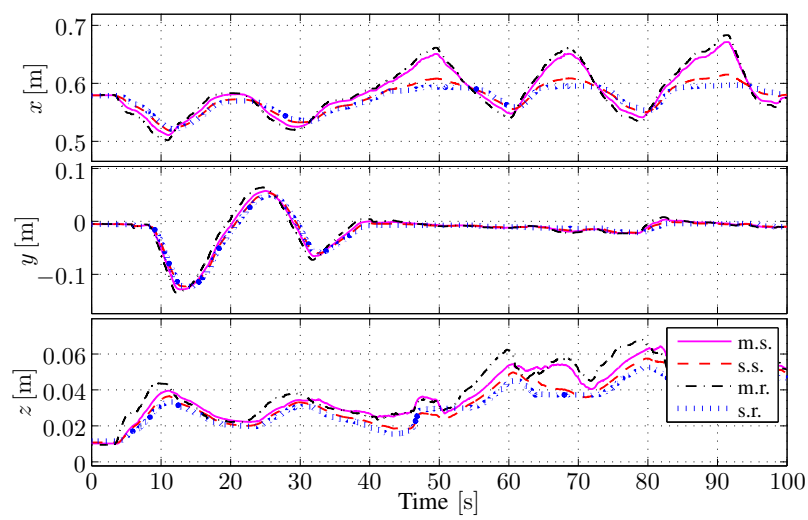

(a) Position

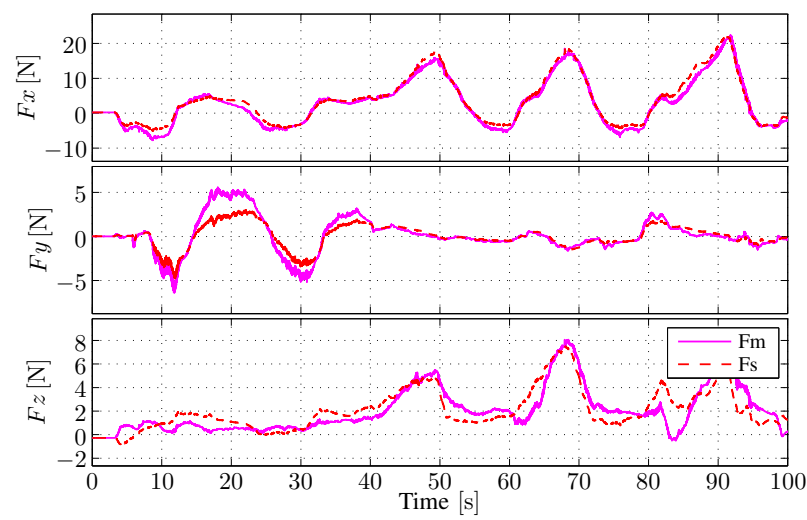

(b) Force

Fig. 5. Teleoperation without virtual fixture: Free space movement (Time $<30 \mathrm{~s}$ ) and contact (Time $>30 \mathrm{~s}$ ).



(a) Position

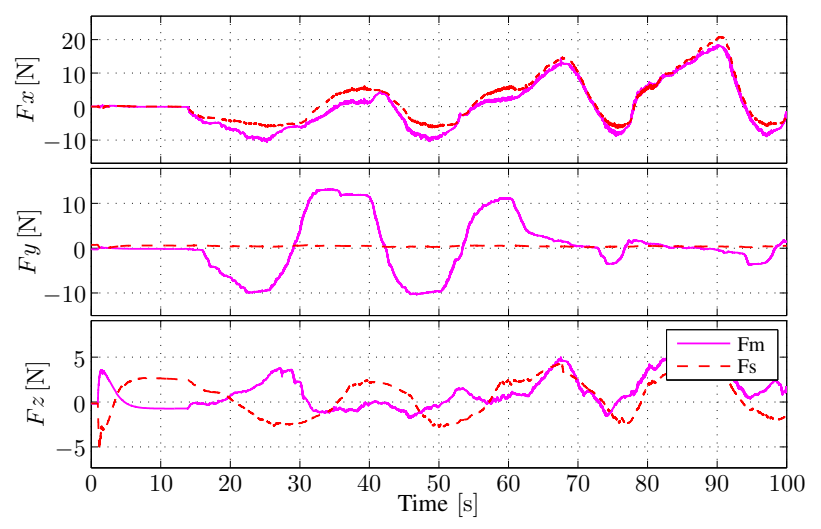

(b) Force

Fig. 6. Teleoperation with virtual fixture. Free space movement (Time $<50 \mathrm{~s}$ ) and contact (Time $>50 \mathrm{~s})$.

\section{REFERENCES}

[1] W. Meeussen, M. Wise, S. Glaser, S. Chitta, C. McGann, P. Mihelich, E. Marder-Eppstein, M. Muja, V. Eruhimov, T. Foote, J. Hsu, R. B. Rusu, B. Marthi, G. Bradski, K. Konolige, B. Gerkey, and E. Berger, "Autonomous door opening and plugging in with a personal robot," in IEEE Proc. of the Int. Conf. on Robotics and Automation, 2010, pp. $729-736$.

[2] M. Fuchs, C. Borst, P. R. Giordano, A. Baumann, E. Kraemer, J. Langwald, R. Gruber, N. Seitz, G. Plank, K. Kunze, R. Burger, F. Schmidt, T. Wimboeck, and G. Hirzinger, "Rollin'Justin-Design considerations and realization of a mobile platform for a humanoid upper body," in IEEE Proc. of the Int. Conf. on Robotics and Automation. IEEE, 2009, pp. 4131-4137.

[3] R. Bauernschmitt, E. U. Schirmbeck, A. Knoll, H. Mayer, I. Nagy, N. Wessel, S. M. Wildhirt, and R. Lange, "Towards robotic heart surgery: Introduction of autonomous procedures into an experimental surgical telemanipulator," International Journal of Medical Robotics and Computer Assisted Surgery, vol. 01, no. 03, p. 74, 2005.

[4] K.-H. Seo and J.-J. Lee, "The development of two mobile gait rehabilitation systems." IEEE transactions on neural systems and rehabilitation engineering, vol. 17, no. 2, pp. 156-66, Apr. 2009.

[5] J. Ren, R. V. Patel, K. a. McIsaac, G. Guiraudon, and T. M. Peters, "Dynamic 3-D virtual fixtures for minimally invasive beating heart procedures." IEEE transactions on medical imaging, vol. 27, no. 8, pp. 1061-70, Aug. 2008.

[6] B. Bauml, F. Schmidt, T. Wimbock, O. Birbach, A. Dietrich, M. Fuchs, W. Friedl, U. Frese, C. Borst, M. Grebenstein, O. Eiberger, and G. Hirzinger, "Catching flying balls and preparing coffee: Humanoid Rollin'Justin performs dynamic and sensitive tasks," in IEEE Proc. of the Int. Conf. on Robotics and Automation, 2011, pp. 3443-3444.
[7] M. Lawitzky, A. Mortl, and S. Hirche, "Load sharing in human-robot cooperative manipulation," in RO-MAN, 2010, pp. $185-191$.

[8] R. Smits, T. Laet, K. Claes, H. Bruyninckx, and J. Schutter, "iTaSC: a tool for multi-sensor integration in robot manipulation," in Multisensor Fusion and Integration for Intelligent Systems, vol. 2. Springer, 2009, pp. 235-254.

[9] Y. Kobayashi, T. Hoshi, K. Kawamura, and M. Fujie, "Control method for surgical robot to prevent overload at vulnerable tissue," in IEEE Proc. of the Int. Conf. on Robotics and Automation, vol. 1, no. April. IEEE, 2007, pp. 1893-1899.

[10] W. Decre, R. Smits, H. Bruyninckx, and J. De Schutter, "Extending iTaSC to support inequality constraints and non-instantaneous task specification," in IEEE Proc. of the Int. Conf. on Robotics and Automation. IEEE, May 2009, pp. 964-971.

[11] J. De Schutter, T. De Laet, J. Rutgeerts, W. Decre, R. Smits, E. Aertbelien, K. Claes, and H. Bruyninckx, "Constraint-based Task Specification and Estimation for Sensor-Based Robot Systems in the Presence of Geometric Uncertainty," The International Journal of Robotics Research, vol. 26, no. 5, pp. 433-455, May 2007.

[12] J. De Schutter, J. Rutgeerts, E. Aertbelien, F. De Groote, T. De Laet, T. Lefebvre, W. Verdonck, and H. Bruyninckx, "Unified constraintbased task specification for complex sensor-based robot systems," in IEEE Proc. of the Int. Conf. on Robotics and Automation. IEEE, 2005, pp. 3607-3612.

[13] O. Khatib, "A unified approach for motion and force control of robot manipulators: The operational space formulation," IEEE Journal of Robotics and Automation, vol. 3, no. 1, pp. 43-53, 1987.

[14] "ROS, the Robot Operating System," www.ros.org.

[15] H. Bruyninckx, P. Soetens, and B. Koninckx, "The Real-Time Motion Control Core of the Orocos Project," in IEEE Proc. of the Int. Conf. on Robotics and Automation, 2003, pp. 2766-2771. 